\title{
Regulation of glucagon secretion by glucose transporter type 2 (glut2) and astrocyte- dependent glucose sensors
}

\author{
Nell Marty, ${ }^{1}$ Michel Dallaporta, ${ }^{1}$ Marc Foretz,, ${ }^{1}$ Martine Emery, ${ }^{1}$ David Tarussio, ${ }^{1}$ \\ Isabelle Bady, ${ }^{1}$ Christophe Binnert, ${ }^{1}$ Friedrich Beermann, ${ }^{2}$ and Bernard Thorens ${ }^{1}$ \\ ${ }^{1}$ Institute of Physiology and Center for Integrative Genomics, Lausanne, Switzerland. \\ ${ }^{2}$ Swiss Institute for Experimental Cancer Research, Epalinges, Switzerland.
}

\begin{abstract}
Ripglut1;glut $2^{-/-}$mice have no endogenous glucose transporter type 2 (glut2) gene expression but rescue glucoseregulated insulin secretion. Control of glucagon plasma levels is, however, abnormal, with fed hyperglucagonemia and insensitivity to physiological hypo- or hyperglycemia, indicating that GLUT2-dependent sensors control glucagon secretion. Here, we evaluated whether these sensors were located centrally and whether GLUT2 was expressed in glial cells or in neurons. We showed that ripglut1;glut $2^{-/}$mice failed to increase plasma glucagon levels following glucoprivation induced either by i.p. or intracerebroventricular 2-deoxy- $D$-glucose injections. This was accompanied by failure of 2-deoxy- $D$-glucose injections to activate c-Fos-like immunoreactivity in the nucleus of the tractus solitarius and the dorsal motor nucleus of the vagus. When glut 2 was expressed by transgenesis in glial cells but not in neurons of ripglut1;glut $2^{-/-}$mice, stimulated glucagon secretion was restored as was c-Fos-like immunoreactive labeling in the brainstem. When ripglut1;glut2-/- mice were backcrossed into the $\mathrm{C} 57 \mathrm{BL} / 6$ genetic background, fed plasma glucagon levels were also elevated due to abnormal autonomic input to the $\alpha$ cells; glucagon secretion was, however, stimulated by hypoglycemic stimuli to levels similar to those in control mice. These studies identify the existence of central glucose sensors requiring glut 2 expression in glial cells and therefore functional coupling between glial cells and neurons. These sensors may be activated at different glycemic levels depending on the genetic background.
\end{abstract}

\section{Introduction}

Glucagon secretion is an immediate response to hypoglycemia, which leads to restoration of normal blood glucose levels by stimulating hepatic glucose production. The normal regulation of this hormone secretion by low glucose becomes progressively impaired in type 1 and some type 2 diabetic patients, and the ensuing risk of developing severe hypoglycemia represents a major obstacle to efficient insulin treatment of these diseases $(1,2)$.

The physiological mechanisms controlling glucagon secretion and how they become deregulated in diabetes are far from being elucidated. Experimental studies in animals and in humans have provided evidence for multiple systems controlling glucagon secretion. First, $\alpha$ cells may directly respond to changes in glucose concentrations $(3,4)$, and they express genes associated with glucose sensing such as glucokinase (5) and the Kir6.2 and SUR1 (6) subunits of the ATP-dependent $\mathrm{K}+\left(\mathrm{K}_{\mathrm{ATP}}\right)$ channel but not glucose transporter type 2 (GLUT2) (7). A second level of control of glucagon secretion is by intraislet insulin levels $(8,9)$. Indeed, insulin is a negative regulator of glucagon secretion, and suppression of insulin secretion by low glucose relieves this inhibition. A major control of $\alpha$ cells' secretory activity, however, occurs through both the sympathetic and parasympathetic

Nonstandard abbreviations used: c-FLI, c-Fos-like immunoreactive; 2-DG, 2-deoxy- $D$-glucose; DMNX, dorsal motor nucleus of the vagus; GFAP, glial fibrillary acidic protein; GLUT2, glucose transporter type 2; i.c.v., intracerebroventricular; $\mathrm{K}_{\mathrm{ATP}}$, ATP-dependent $\mathrm{K}^{+}$; NTS, nucleus of the tractus solitarius; $\mathrm{VMH}$, ventromedial hypothalamic nucleus.

Conflict of interest: The authors have declared that no conflict of interest exists.

Citation for this article: J. Clin. Invest. 115:3545-3553 (2005).

doi:10.1172/JCI26309. branches of the autonomic nervous system and by the sympathoadrenal axis $(10,11)$.

The activation of the autonomic nervous system and sympathoadrenal axis by hypoglycemia depends on glucose sensing mechanisms, which are yet poorly defined but probably reside at several anatomical sites, including the hepatoportal vein area, the brainstem, and the hypothalamus. Hepatoportal vein glucose sensors are linked by hepatic vagal afferents to brainstem nuclei, in particular the nucleus of the tractus solitarius (NTS) and the lateral hypothalamus $(12,13)$. Their role in glucagon secretion is still debated, and they may not be critical for hypoglycemia-induced glucagon secretion; however, their activation by portal glucose injection can suppress the glucagon response to insulin-induced peripheral hypoglycemia $(14,15)$.

The role of hypothalamic nuclei in counterregulation has been explored through lesion studies and pharmacological or genetic interference with glucose detection systems (16). From these studies, the ventromedial hypothalamic nucleus $(\mathrm{VMH})$ appears to play a critical role. Indeed, hypoglycemia-induced glucagon secretion was suppressed by direct $\mathrm{VMH}$ injection of glucose (17) or of $\mathrm{K}_{\mathrm{ATP}}$ channel inhibitors (18). Inactivation of $\mathrm{K}_{\mathrm{ATP}}$ channel in Kir6.2-/- mice also led to impaired glucagon response, which was correlated with a suppression of the glucose-regulated VMH neurons firing activity (19). In addition, glucoprivation by direct injection of 2-deoxy- $D$-glucose (2-DG) in the VMH induced glucagon secretion (20).

Whereas the above data support a role for the $\mathrm{VMH}$ in the control of glucagon secretion, other published data indicate that glucosesensing units located in the brainstem play an equally critical role in the physiological control of glucagon secretion. For instance, when the cerebral acqueduct is obstructed, 2-DG induces a glucoregulatory response only when injected in the fourth but not the 
A
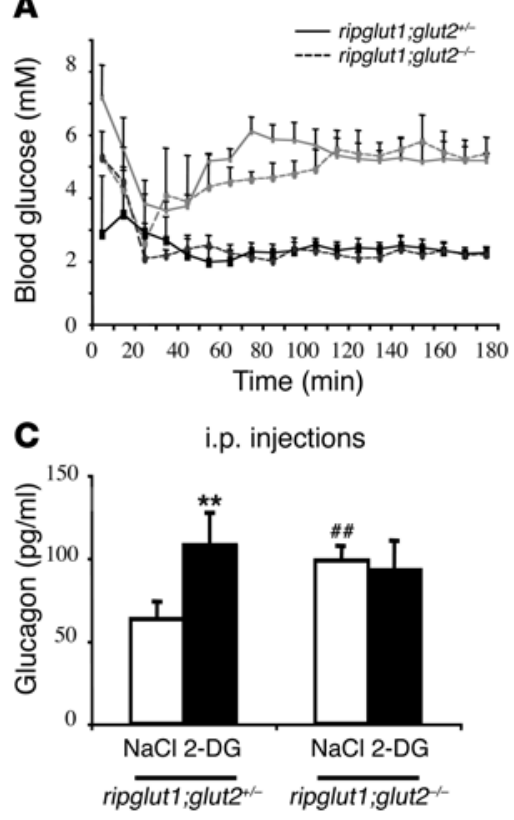

B
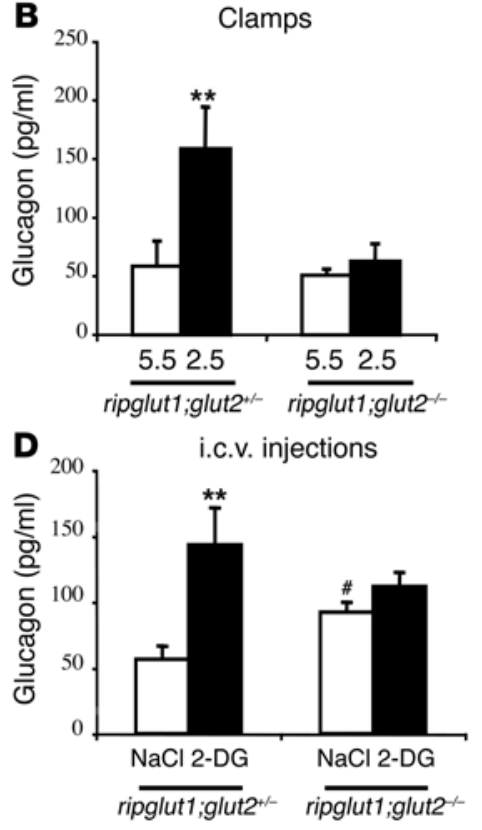

Figure 1

Suppression of glucagon secretion in response to physiological hypoglycemia or cellular glucoprivation in ripglut1; glut2-/- mice. (A) Glycemic profiles during use of normoglycemic or hypoglycemic clamps in ripglut1;glut2+and ripglut1; glut2 $2^{-1-}$ mice. (B) Plasma glucagon levels measured at the end of the clamp experiment. Hypoglycemia induced an approximately 3-fold increase in plasma glucagon in control mice but no increase in ripglut $1 ;$ glut2 $2^{-/}$mice. (C) Glucagon levels 30 minutes after i.p. injection of $\mathrm{NaCl}$ or 2-DG. 2-DG induced an approximately 1.7-fold increase in plasma glucagon in control mice but no increase in glut2null mice. (D) Glucagon levels measured 30 minutes following i.c.v. injection of $\mathrm{NaCl}$ or 2-DG. 2-DG induced a 2.5 -fold increase in plasma glucagon in control mice and no increase in ripglut1;glut2 ${ }^{-/}$mice. (B and $\mathbf{D}$ ) Data are indicated as mean \pm SD; $n=6-10$ for each data point. (C) Data are indicated as mean \pm SEM of 3 experiments, each performed with 6-8 mice. ${ }^{* \star} P<0.01$ for comparison between $\mathrm{NaCl}$ - and 2-DG-injected groups. ${ }^{*} P<0.05$ and $\# P<0.01$ for comparison between $\mathrm{NaCl}$-injected control and ripglut1; glut2--- groups (Student's $t$ test). lateral ventricle (21). Also, localization of 2-DG-activated neurons by c-Fos immunostaining (22) showed that the NTS, the dorsal motor nucleus of the vagus (DMNX), and the A1, A1/C1, and C1 groups of catecholaminergic neurons of the brainstem were particularly responsive to this glucoprivic challenge. In addition, whereas injections of 5-thioglucose in different nuclei of the hypothalamus failed to induce a glucoregulatory response, strong responses were obtained by injections of this antimetabolite into the NTS and the medullary regions containing the $\mathrm{A} 1$ and $\mathrm{C} 1$ catecholaminergic neurons (23). These catecholaminergic neurons project to different sites of the hypothalamus to control counterregulation but also feeding (23-25). The NTS has also been demonstrated to be sensitive to small variations in blood glucose concentrations, a mechanism that requires the function of the $\mathrm{K}_{\text {ATP }}$ channel $(26,27)$.

Together, these data suggest that the glucose-sensing systems involved in controlling glucagon secretion form a highly interactive network linked by nerve connections; in this network, the brainstem may have a critical role for physiological hypoglycemia sensing.

The cells and molecules involved in glucose sensing and counterregulation have not yet been identified. This is due to a lack of molecular markers that could be unambiguously associated with the physiological response to hypoglycemia and that could be used to identify the responsive cells. The $\mathrm{K}_{\mathrm{ATP}}$ channel is involved in central glucose sensing $(18,19)$; however, the Kir6.2 subunit is expressed at a high level in all brain areas (28) and thus cannot provide precise markers for glucose-sensitive cells.

In a previous study, we showed that ripglut1;glut2-/- mice, which have an inactivated glut2 gene but normal glucose-regulated insulin secretion due to transgenic expression of GLUT1 in their $\beta$-cells (29), have abnormal regulation of glucagon plasma levels. This was characterized by fed hyperglucagonemia, which could be normalized by ganglionic blockade, indicating an increased autonomic tone to the $\alpha$ cell, and by suppressed response to moderate hypoglycemic levels $(\sim 2.5 \mathrm{mM})$. However, a strong response could still be induced by deep hypoglycemia $(\leq 1 \mathrm{mM})$. These data indicate that glucagon secretion is under the control of GLUT2-dependent and independent hypoglycemia detection systems that can be activated at different glycemic levels.

Here, we extend these observations by providing evidence that GLUT2-dependent glucose sensors are located centrally, that they are involved in the activation of neurons of the NTS and DMNX, and that GLUT2 needs to be expressed in glial cells for the glucodetection system to function.

\section{Results}

Absence of stimulated glucagon secretion in ripglut1;glut2-/- mice. Ripglut1;glut2 ${ }^{-/-}$mice have an inactivated endogenous glut 2 gene and express transgenic glut 1 in their $\beta$ cells. This restores normal glucose-induced insulin secretion and allows the mice to live and reproduce normally. These mice have normal body weight and normal glycemia in the fed state; their glycemia is approximately $1 \mathrm{mM}$ lower than that of control mice in the fasted state. They have been described in detail in previous studies $(29,30)$.

We first performed hyperinsulinemic clamping in ripglut $1 ; g l u t 2^{-/-}$ mice and their control littermates, and we assessed plasma glucagon levels at the end of the experiments. Figure 1A shows the glycemic profiles of mice clamped at approximately $5.5 \mathrm{mM}$ or approximately $2.5 \mathrm{mM}$ glucose for 3 hours. The plasma glucagon levels determined at the end of the clamp periods were markedly increased by hypoglycemia in the control mice but not in ripglut 1 ;glut2-/- mice (Figure $1 \mathrm{~B}$ ), in agreement with previous studies (29). Since GLUT2 is not expressed in pancreatic $\alpha$ cells (7) and the control by glucose of insulin secretion is normal in ripglut 1 ;glut2 $2^{-/}$mice (30), this indicated that extrapancreatic, GLUT2-dependent, glucose-sensing mechanisms were involved in hypoglycemia-induced glucagon secretion.

Next, we evaluated the response to a glucoprivic signal induced by administration of 2-DG. As our previous studies indicated that glucagon secretion in mutant mice could still be induced by deep hypoglycemia, we first selected a dose of 2-DG that could assist us in discriminating between the responses of control and mutant mice. We tested i.p. doses of $75,100,150,200$, and $400 \mathrm{mg} / \mathrm{kg}$ and 
A

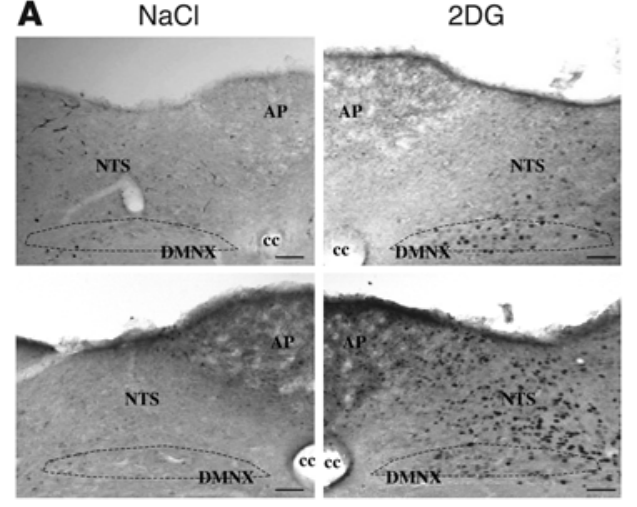

B
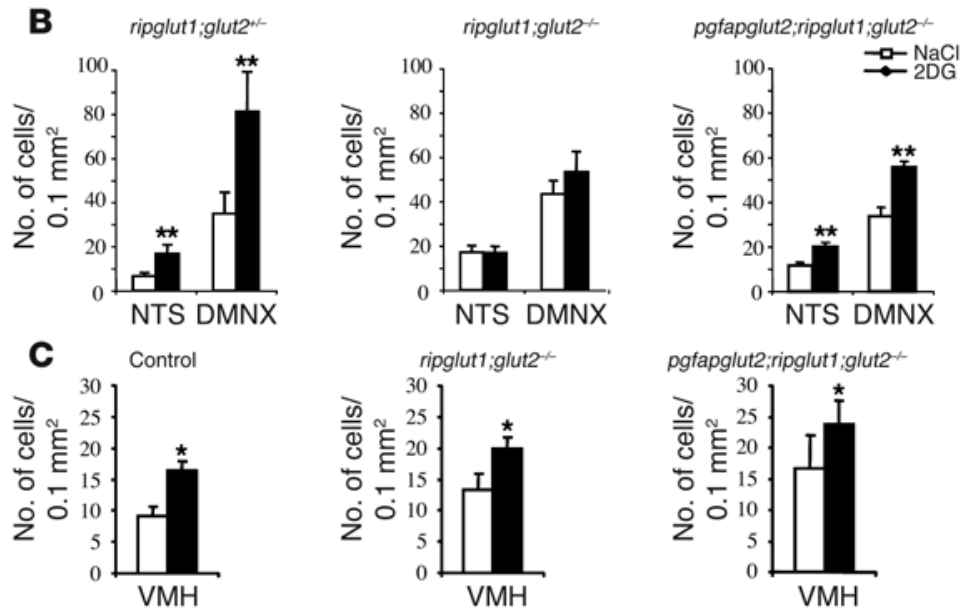

Figure 2

c-FLI cells in the NTS, the DMNX, and the VMH following 2-DG injections. Mice were injected i.p. with NaCl or 2-DG, and tissues were processed 2 hours later for c-FLI immunohistochemistry. (A) Representative photos from c-FLI-positive cells at 2 different levels of the NTS and DMNX of ripglut1; glut2+/- mice after i.p. injection of $\mathrm{NaCl}$ or 2-DG. The positions of the sections were between -7.64 and $-7.48 \mathrm{~mm}$ relative to the bregma. Scale bars: $100 \mu \mathrm{m}$. AP, area postrema; cc, central canal. (B) c-FLI-positive cells in the NTS and DMNX of ripglut1; glut2 ${ }^{+-}$, ripglut1; glut2-/-, and pgfapglut2;ripglut1; glut2-/- mice after i.p. injection of $\mathrm{NaCl}$ or 2-DG. In glut2-null mice, there is no increase in the number of c-FLI-positive cells after 2-DG administration. Transgenic expression of GLUT2 in astrocytes restored the sensitivity of the NTS and DMNX to i.p. 2-DG. (C) c-FLIpositive cells in the $\mathrm{VMH}$ of control, ripglut1; glut2--, and pgfapglut2;ripglut1; glut2--- mice after i.p. injection of $\mathrm{NaCl}$ or 2-DG. Data are indicated as mean \pm SD; $n=6-8$ mice for each data point. ${ }^{*} P<0.05$, ${ }^{\star *} P<0.01$ for comparison between NaCl- and 2-DG-injected groups (Student's $t$ test).

selected a dose of $150 \mathrm{mg} / \mathrm{kg}$ for all subsequent i.p. injection experiments. The optimal time for plasma glucagon level measurement after injection of 2-DG was also evaluated in control mice. The strongest response was found at 30 minutes; it was still almost as high at 60 minutes and reduced at 120 minutes. Figure $1 \mathrm{C}$ shows the plasma glucagon levels in fed control and mutant mice 30 minutes after injection of saline or 2-DG. In control mice, 2-DG induced a significant increase in plasma glucagon levels whereas in ripglut1;glut2-/- mice, basal plasma glucagon levels were higher than in their littermates, as previously reported for fed mice (29), but were not increased by $2-D G$ injection. This suggests that the 2-DG glucoprivic signal was not detected in the absence of GLUT2.

Next, we evaluated whether central GLUT2-dependent glucose sensors were also involved in the 2 -DG response. To perform these analyses, we implanted mice with cannulas in the lateral ventricle. Correct positioning of the cannulas was verified at the end of the experiments by histological analysis, and only results from mice with correctly implanted cannulas were considered. Figure 1D shows that plasma glucagon levels 30 minutes after $2-D G(1 \mathrm{mg} /$ mouse) administration were increased only in control but not in mutant mice. This indicates that the central 2-DG-sensitive units controlling glucagon secretion are GLUT2-dependent.

c-Fos-like immunoreactivity in control and ripglut1;glut2-/- mice. To evaluate whether the absence of glucagon response to 2-DG injections in mutant mice was correlated with a change in the activity of certain glucose-sensitive brainstem areas, we chose to analyze the number of c-Fos-like immunoreactive-positive (c-FLI-positive) cells in the NTS and the DMNX, 2 regions of the dorsal vagal complex intimately linked to afferent signals from the gut and also connected to afferent and efferent signals from different nuclei of the hypothalamus.

The data in Figure 2, A and B, show that, following i.p. 2-DG injection, there was a marked increase in c-FLI-positive cells in the NTS and the DMNX of control (ripglut1;glut2+/-) mice, which was not observed in mutant mice (Figure 2B). In the NTS, the number of c-FLI-positive cells in the basal state was, however, similar to that in 2-DG-treated control mice. These data thus indicated that these brainstem structures were sensitive to the glucoprivic stress induced by i.p. 2-DG and that the response required the presence of GLUT2. In the absence of GLUT2, there was permanent activation of the NTS.

The VMH has been previously linked to regulation of glucagon secretion in response to hypoglycemia. We therefore assessed the c-FLI-positive cells in this hypothalamic structure in control and ripglut1;glut2 $2^{-1-}$ mice following i.p. 2-DG injections. Figure $2 \mathrm{C}$ shows that this glucoprivic signal induced a similar increase in c-FLI-positive cells in the VMH of the control and mutant mice, indicating that activation of this structure is independent of GLUT2 expression. The relatively low increase in c-FLI-positive cells in the VMH is probably due to the relatively low dose of 2-DG used in our experiments as compared with similar studies performed in the rat, in which the usual dose is $400 \mathrm{mg} / \mathrm{kg}$.

GLUT2 expression in glial cells restores glucagon secretion. Certain neurons can increase or decrease their firing rates in response to variations in extracellular glucose concentrations $(31,32)$. It is not known, however, whether glucose is directly metabolized by neurons to regulate their firing activity or first taken up by astrocytes, then catabolized to lactate, which is then transferred to neurons for ATP production and regulation of their electrical activity (33).

To address this question, we prepared transgenic mice expressing GLUT2 either under the synapsin or under the glial fibrillary acidic protein (GFAP) promoters for expression in neurons or astrocytes, respectively. Figure 3A shows the construct used for generating the gfapglut2 mice. This construct was injected into oocytes of B6D2F1 mice fertilized by ripglut1;glut $2^{-/-}$males. The founders were then backcrossed into ripglut 1 ;glut2 $2^{-1-}$ mice to obtain gfapglut2;ripglut1;glut2-/- mice. Figure 3B shows the presence of the transgene, detected by Southern blot analysis as a $3.8-\mathrm{kb}$ 
A
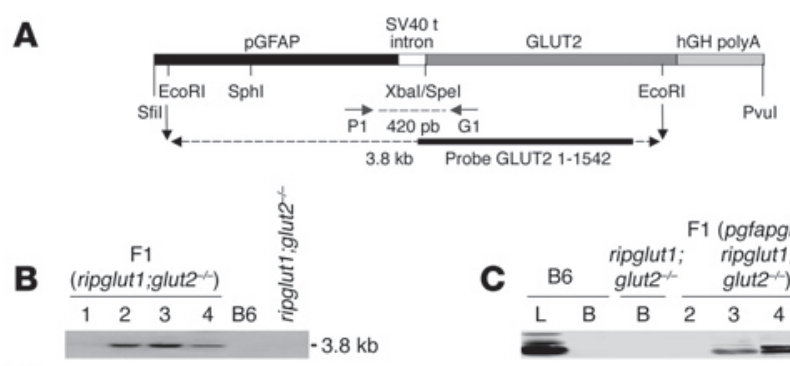

D

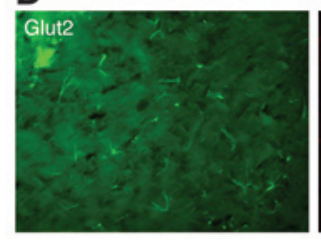

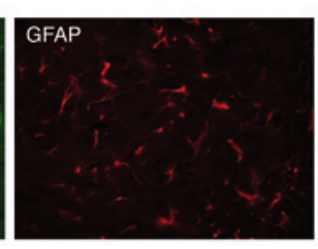
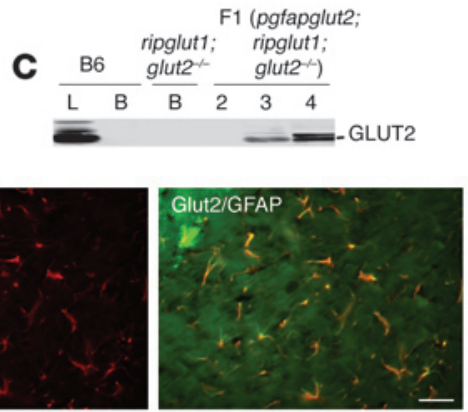

\section{Figure 3}

Expression of GLUT2 in astrocytes of ripglut1;glut2-/- mice restores glucagon secretion in response to physiological hypoglycemia or cellular glucoprivation. (A) Scheme of the GLUT2 transgene. (B) Southern blot detection of the 3.8-kb EcoRI transgene fragment in the genomic DNA of $\mathrm{F} 1$ mice from 3 of the 4 lines of transgenic mice. (C) Western blot detection of GLUT2 in whole-brain membranes of pgfapglut2;ripglut1;glut2-1mice. GLUT2 is detected in the brains (B) of lines 3 and 4. GLUT2 expression in liver (L) of B6 mice is presented as a control. GLUT2 is not detectable by Western blot analysis in the brains of B6 or ripglut1; glut2-/- mice, as expected from its very low abundance. (D) Immunofluorescence detection in the brains of mouse transgenic line 4 of GLUT2 and GFAP. Overlay of both types of staining shows expression of transgenic GLUT2 in glial cells. Scale bar: $50 \mu \mathrm{m}$.
DNA fragment, in the different transgenic lines. Expression of GLUT2 in the brains of these mice was ascertained by Western blot analysis. Figure 3C shows that GLUT2 was not detected in whole-brain membrane preparations from control mice, as expected from its very low level of expression in restricted subpopulations of neurons or astrocytes (34). In contrast, GLUT2 was detected in transgenic lines 3 and 4 . Immunofluorescence microscopy showed a good colocalization of transgenic GLUT2 with the glial cell marker GFAP (Figure 3D). Line 4 was selected for subsequent physiological analysis.

The glucagon response during a hypoglycemic clamp or following i.p. injection of 2-DG was then measured in gfapglut2;ripglut1;glut2 $2^{-/-}$mice and in their appropriate glut2 $2^{-/-}$control littermates. Figure $4 \mathrm{~A}$ shows the plasma glucagon levels measured at the end of hypo- $(\sim 2.5 \mathrm{mM})$ or euglycemic $(\sim 5.5 \mathrm{mM})$ clamping. Transgenic expression of GLUT2 in glial cells restored the plasma glucagon response to hypoglycemia. Similarly, following i.p. injections of 2-DG, the plasma glucagon response was restored in gfapglut2;ripglut1;glut2 $2^{-/-}$mice (Figure 4B).

We next evaluated whether these rescued mice had restored activation of NTS and DMNX neurons following i.p. 2-DG injections. As shown in Figure 2B, the number of c-FLI-positive cells in the NTS of gfapglut2;ripglut1;glut2-/mice was increased following $2-\mathrm{DG}$ injection. The difference was in part due to a decrease in the c-FLI-positive cells in the basal state. In the DMNX, GLUT2 reexpression also restored a sensitivity to $2-\mathrm{DG}$-induced activation of c-FLI-positive cells. In the $\mathrm{VMH}$, no change in the increase in c-FLI-positive cells was observed in the gfapglut2;ripglut1;glut2-/- mice (Figure 2C).

Thus, glial cell reexpression of GLUT2 restored glucagon response to hypoglycemia and glucoprivic signals and activation of NTS and DMNX cells by 2-DG injections.

GLUT2 expression in neurons has no effect on glucagon secretion. As a further control for the role of GLUT2 in glial cells in the glucagon response, we also generated mice with transgenic expression of GLUT2 in neurons under the control of the synapsin promoter. The vector used for the preparation of transgenic mice is shown in Figure 5A. The transgenic and breeding strategy was similar to that used for glial cell reexpression of GLUT2. Expression of GLUT2 in the brains of the synapsinglut2;ripglut1;glut2-/- transgenic mice was detected by Western and Northern blot analysis (Figure 5, B and C). Expression of the GLUT2 mRNA was found in wholebrain extracts but also in extracts from the NTS and hypothalamus. GLUT2 protein was also detected in whole-brain extracts from the transgenic mice. For subsequent analysis, lines 1 and 3 were selected. The glucagon response to intracerebroventricular (i.c.v.) 2-DG injections in synapsinglut2;ripglut1;glut2-/- (line 1) is shown in Figure 5D. The ripglut1;glut $2^{+/-}$control littermates presented normal glucagon responses. The ripglut 1 ;glut $2^{-/-}$control littermates failed to increase their plasma glucagon levels, as described above. Importantly, no glucagon response was observed in the synapsinglut2;ripglut1;glut2-mice. Identical results were found with line 3 (not shown).

Together, the above data suggest that the glucagon response to 2-DG or to hypoglycemic clamps depends on glucose-sensing units consisting of metabolically coupled, GLUT2-expressing glial cells and neurons.

Role of glut2-dependent glucose sensors in different genetic backgrounds. The ripglut1;glut2 $2^{--}$mice studied above were in a mixed genetic background originating from Sv129, C57BL/6, and DBA2 mice. To evaluate the
A

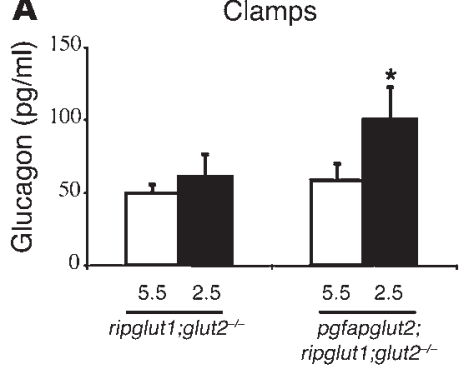

B

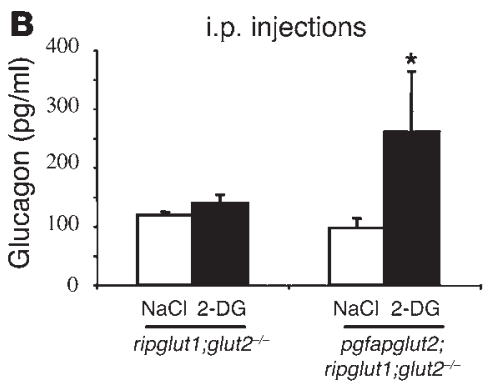

Figure 4

Restoration of glucagon secretion in response to physiological hypoglycemia or cellular glucoprivation in pgfapglut2;ripglut1;glut2-/- mice. (A) ripglut1;glut2-/and pgfapglut2;ripglut1; glut2--- mice were clamped for 3 hours at low $(\sim 2.5 \mathrm{mM})$ or euglycemic $(\sim 5.5 \mathrm{mM})$ levels and plasma glucagon concentrations measured at the end of clamping. Hypoglycemia did not increase plasma glucagon in ripglut 1 ; glut2 ${ }^{-/-}$mice but induced a 1.7-fold increase in plasma glucagon in pgfapglut2;ripglut1; glut2 ${ }^{-/-}$mice. (B) Plasma glucagon levels measured 30 minutes after i.p. injection of $\mathrm{NaCl}$ or 2-DG. 2-DG induced a 2.7-fold increase in plasma glucagon in pgfapglut2;ripglut1; glut2-/- mice. Data are indicated as mean $\pm \mathrm{SD} ; n=6-10 .{ }^{*} P<0.05$ for comparison between euglycemic and hypoglycemic clamps or $\mathrm{NaCl}$ - and 2-DG-injected groups (Student's $t$ test). 
A

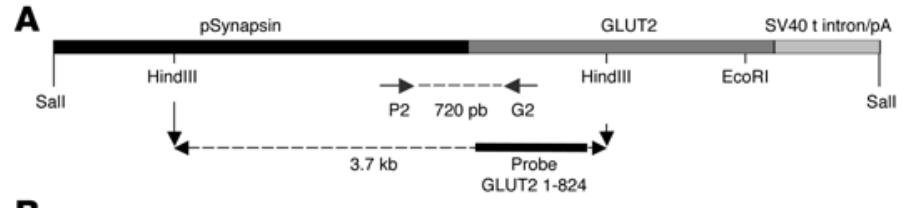

B

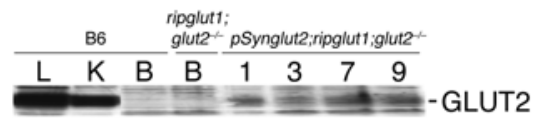

C F1 (pSynglut2;ripglut1; ;lut2

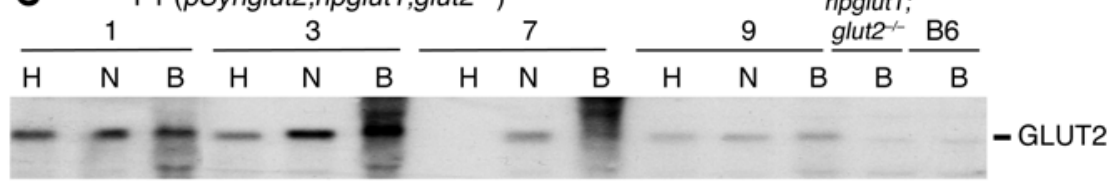

D

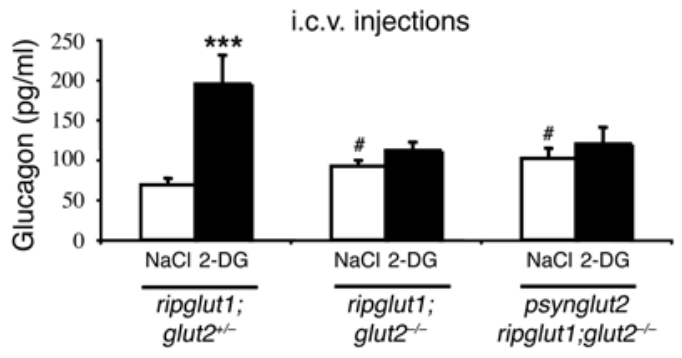

Figure 5

Expression of GLUT2 in neurons of ripglut1; glut2-/mice did not restore glucagon secretion in response to physiological hypoglycemia or cellular glucoprivation. (A) Schematic representation of the GLUT2 transgene. (B) Western blot analysis of GLUT2 expression in total brain membrane of psynglut2;ripglut1; glut2--- transgenic mice. K, kidney. (C) Northern blot analysis of GLUT2 mRNA in hypothalamus $(\mathrm{H}), \mathrm{NTS}(\mathrm{N})$, and whole brains of psynglut2;ripglut1; glut2--- mice, lines $1,3,7$, and 9. Higher expression of the transgene mRNA was found in lines 1 and 3; these lines were selected for subsequent physiological analysis. (D) Plasma glucagon levels measured 30 minutes after i.c.v. injection of $\mathrm{NaCl}$ or 2-DG. Whereas 2-DG induced a glucagon response in ripglut1; glut2+-- mice, no response was observed in ripglut1; glut $2^{-/-}$or in psynglut2;ripglut1;glut2-/- (line 1) mice. The same results were obtained with line 3 mice. Data are indicated as mean $\pm \mathrm{SD} ; n=6-8$. ${ }^{* * *} P<0.005$ for comparison between $\mathrm{NaCl}$ - and 2-DG-injected groups; $\# P<0.05$ for comparison between $\mathrm{NaCl}$-injected control (ripglut1; glut2+-) and ripglut1; glut2 ${ }^{-/}$or psynglut2;ripglut1; glut2 $^{-1-}$ groups (Student's $t$ test). role of the genetic background in the observed glycemic response, we first determined whether the glucagon response to 2-DG was present in Sv129, B6D2F1 (C57BL/6xDBA2F1), or Sv129xB6D2F1 mice. Following i.c.v. injections of saline, the basal glucagon levels were similar in each of these strains $(44 \pm 7 \mathrm{pg} / \mathrm{ml}, 57 \pm 7 \mathrm{pg} / \mathrm{ml}, 50 \pm 4 \mathrm{pg} / \mathrm{ml}$, respectively) (Figure 6,A-C). Administration of 2-DG induced a marked increase in plasma glucagon levels although the responses were clearly of different magnitude in the various strains tested: $102 \pm 37 \mathrm{pg} / \mathrm{ml}$, $195 \pm 6 \mathrm{pg} / \mathrm{ml}$, and $294 \pm 70 \mathrm{pg} / \mathrm{ml}$ for the Sv129, B6D2F1, and Sv129xB6D2F1 mice, respectively. Nevertheless, this lent further support for defective glucagon response in ripglut1;glut $2^{-/-}$mice being caused by absence of GLUT2 expression.

Next, we studied the glucagon levels in the basal state and in response to hypoglycemia and glucoprivic signals in ripglut1;glut2-/mice backcrossed for 7 generations in the C57BL/ 6 background. Figure 7A shows that the glucagon level was higher in fed male ripglut1;glut2-/- (B6) mice as compared with control mice, as found in mutant mice with mixed genetic backgrounds. To evaluate whether this was caused by an increase in autonomic tone to $\alpha$ cells, we injected control or mutant female mice in the fed state with saline or chlorisondamine, a ganglionic blocker, and measured plasma glucagon levels 30 minutes later. As shown in Figure $7 \mathrm{~B}$, chlorisondamine treatment led to a decrease of the plasma glucagon to the level found in the control mice and did not affect the glucagonemia of control mice. This indicated that in the ripglut1;glut2-/- (B6) mice, there was an increase in autonomic tone to the $\alpha$ cells that increased glucagon secretion (29).

To evaluate the stimulation of glucagon secretion by hypoglycemia, control and ripglut1;glut2-/- (B6) mice were clamped at approximately $5.5 \mathrm{mM}$ and approximately $2.5 \mathrm{mM}$ glucose for 3 hours. As shown in Figure 7C, the glucagon plasma levels were increased to similar levels in both control and mutant mice by hypoglycemia.

Finally, the glucagon response to injections of saline or 2-DG was measured in control and glut2-null mice. Figure 7D shows that i.p. injections of 2-DG induced an approximately 5-fold increase in plasma glucagon in $\mathrm{C} 57 \mathrm{BL} / 6$ mice and an approximately 3 -fold increase in ripglut1;glut2-/-(B6) mice; the plasma levels in both types of mice were, however, similar. Figure 7E shows the plasma glucagon levels after i.c.v. saline or 2-DG injections. As for the i.p. injections, 2-DG induced an approximately 5-fold increase in plasma glucagon in C57BL/6 mice and an approximately 3 -fold increase in ripglut1;glut2-/-(B6) mice, and the plasma concentrations reached the same levels in both control and mutant mice. Thus, in the absence of GLUT2 expression, glut2-null mice in mixed or B6 genetic backgrounds showed the same defect in basal glucagon regulation caused by increased autonomic tone to the $\alpha$ cells. However, stimulated secretion in the ripglut1;glut2--(B6) mice reached the same levels as in the C57BL/ 6 control mice, although the fold stimulation was lower.

\section{Discussion}

Here, we show that fed glucagonemia and glucagon secretion in response to hypoglycemia or a glucoprivic signal were impaired in mice with inactivation of the glut2 gene. We provided evidence that these deregulations were caused by inactivation of centrally located glucose sensors that require expression of GLUT2 in glial cells and control the activation of the NTS and DMNX. The importance of these sensors on stimulated secretion was, however, influenced by the genetic background.

Our experiments were performed on mice with heterogeneous genetic backgrounds issued from Sv129, C57BL/6, and DBA2 strains. In these mice, inactivation of the glut2 gene led to fed hyperglucagonemia and suppressed glucagon secretory response to hypoglycemia or to i.p. or i.c.v. 2-DG injections. Abnormal regulation of glucagon secretion is probably not caused by the genetic contribution of 1 of the strains used since, when tested individually, mice from each strain showed the same fed glucagonemia and a marked increase in plasma glucagon levels in response to 2-DG. Thus, impaired glucagon response was most likely caused by the absence of GLUT2 expression. 
A

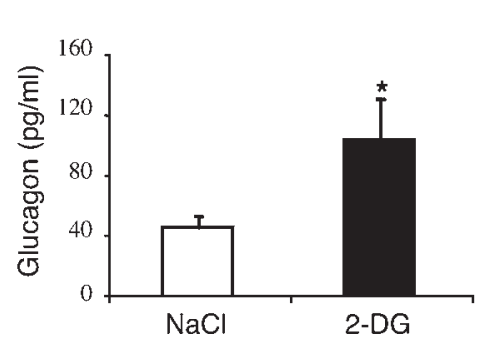

B

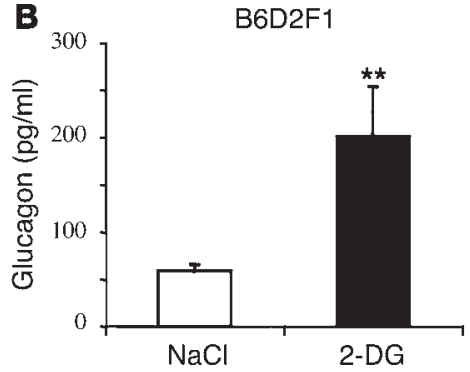

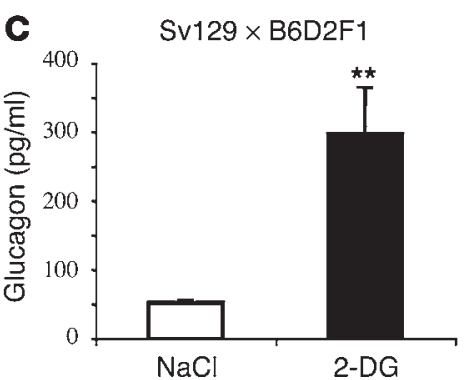

Figure 6

Normal glucagon secretion in response to i.c.v. injection of 2-DG in Sv129, B6D2F1, and Sv129xB6D2F1 mice. Plasma glucagon levels measured 30 minutes after i.c.v. injection of $\mathrm{NaCl}$ or 2-DG in Sv129 (A), B6D2F1 (B), and Sv129xB6D2F1 (C) mice. 2-DG induced a strong increase in plasma glucagon levels in each mouse strain. Data are indicated as mean $\pm \mathrm{SD} ; n=6-7$ for each data point. ${ }^{*} P<0.05$ and ${ }^{* *} P<0.01$ for comparison between $\mathrm{NaCl}$ - and 2-DG-injected groups (Student's $t$ test).

The site or sites of hypoglycemia or glucoprivic signal detection leading to glucagon secretion have not been formally identified. It is, however, well established from multiple previous studies that the CNS is involved in the counterregulatory response to hypoglycemia. One site that is sensitive to both central and peripheral glucoprivic signals and to insulin-induced hypoglycemia is the NTS, a structure where GLUT2 has been reported to be present $(34,35)$, and where neurons directly sensitive to small variations in blood glucose concentrations are located (26). Neurons from the NTS send projections to the hypothalamic nuclei, in particular to the PVN and the LH, but also to the DMNX and are connected to the pancreas (36). In the brainstem, other regions are also known to be sensitive to the glucoprivic signal generated by $2-\mathrm{DG}$ injections, such as the $\mathrm{A} 1$ and $\mathrm{C} 1$ catecholaminergic neurons located in the ventrolateral medulla, which are involved in the glucoregulatory response to glucoprivation $(22,23)$.

In our present study, the suppressed glucagon response in glut2-null mice was associated with a loss of c-FLI responsiveness in the NTS and DMNX, suggesting that these regions are indeed activated by the GLUT2-dependent sensors controlling counterregulation. The glucose-sensing cells expressing GLUT2 are, however, not yet formally identified. By light and electron microscopic immunolocalization, GLUT2 was found to be expressed variously in astrocytes, in neurons, and in ependymal cells (34, 37-39). To directly address the contribution of GLUT2 in astrocytes and in neurons, we expressed GLUT2 either in glial cells or in neurons in transgenic mice and backcrossed them into the ripglut1;glut $2^{-/-}$background. Our data showed that expression of GLUT2 in glial cells restored the glucagon response to hypogylcemia and to i.p. or i.c.v. 2-DG injection. Furthermore, this was associated with a restoration of c-Fos staining in the NTS and the DMNX. No such restoration of glucagon secretion could be found when GLUT2 was expressed by transgenesis in neurons. This, therefore, implies that GLUT2 needs to be expressed in astrocytes for the correct glucose sensing. These data are compatible with reports by 2 other laboratories showing that methionine sulfoximine (MSO), a glutamine synthetase inhibitor that impairs carbohydrate metabolism specifically in astrocytes, attenuated the glucoprivic activation of NTS neurons (40) or the secretion of insulin and activation of hypothalamic neurons following intracarotid infusion of glucose (41). These observations are thus in agreement with the model of a metabolic coupling between astrocytes and neurons (33). This model suggests that glucose is initially taken up by astrocytes and metabolized to lactate, which is then transported into neurons for ATP generation and control of nerve firing rate. However, it must be pointed out that GLUT2 expression is much higher in transgenic than in control astrocytes. Although this level of overexpression may not be physiological, it must be emphasized that in the $\beta$-cell paradigm of glucose sensing, glucose phosphorylation by glucokinase is the rate-controlling step in signal generation, and the rate of uptake is much greater than the rate of phosphorylation. Thus, the exact level of transgene overexpression is not a critical factor in restoring glucose sensing. Transgenic expression of GLUT2 under the synapsin promoter failed to restore glucagon secretion in response to glucoprivic signals. However, although GLUT2 was expressed at high levels in the hypothalamus and brainstem, we could not formally prove that expression was in the neurons normally expressing this transporter. These negative results nevertheless confirm that the transporter is required for normal glucoprivic signal detection.

One interesting observation was that, when backcrossed in the C57BL/ 6 genetic background, the ripglut 1, glut $2^{-/-}$mice presented a partially different phenotype. This was characterized by high-fed glucagonemia as found in mice with a mixed genetic background but a strong stimulation of glucagon secretion by hypoglycemia and 2-DG injections as in control mice. The high-fed glucagonemia was suppressed by the ganglionic blocker chlorisondamine, indicating the presence of a high autonomic tone to the $\alpha$ cells as in glut2-null mice on a mixed background. However, the stimulated glucagon secretion reached the same level in the mutant as in the C57BL/ 6 control mice. Thus, this suggests that the mechanisms controlling fed glucagonemia and glucagon secretion in response to hypoglycemia may be separate.

Glucagon secretion in response to hypoglycemia is stimulated by direct $\alpha$ cell glucose sensing, by a fall in insulin secretion, and by activation of the autonomic nervous system and of the sympatoadrenal axis. It is thus possible that there is a differential activation of these control systems at defined hypoglycemic levels and that these may change between different strains of mice. This would be analogous to the secretion of insulin in different strains of mice. For instance, C57BL/ 6 and A/J mice show glucose-stimulated insulin secretory responses, which display very different $\mathrm{Km}$ and $\mathrm{Vmax}$ even though they express the same critical proteins involved in glucodetection, such as GLUT2, glucokinase, and other glycolytic 
A
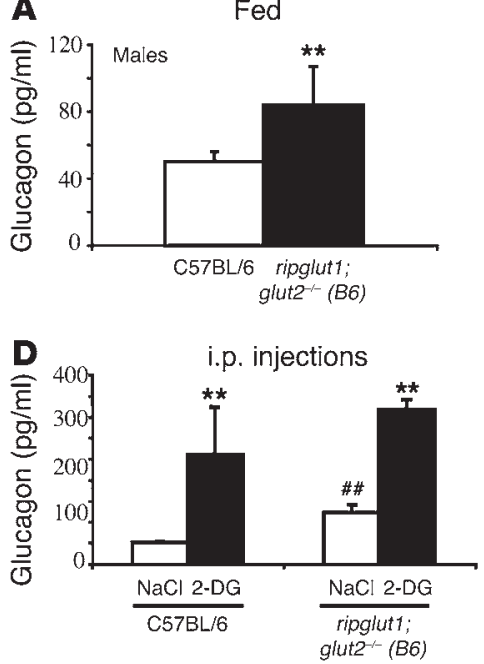
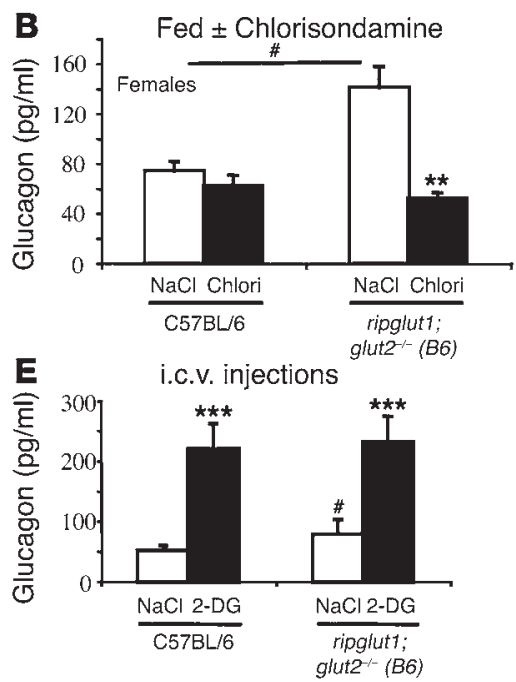

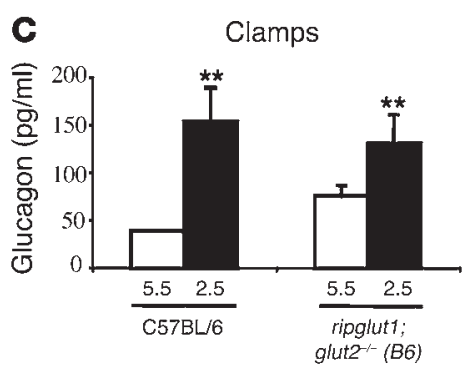

Figure 7

Abnormal glucagonemia in the fed state but normal glucagon secretion in response to hypoglycemia or 2-DG in ripglut1; glut2-/- mice in the C57BL/6 background. (A) Fed glucagon levels were approximately 2-fold higher in male ripglut1;glut2--(B6) than in C57BL/6 mice; (B) Fed glucagon levels were approximately 2 -fold higher in female ripglut1;glut2--(B6) than in C57BL/6 mice. The fed hyperglucagonemia of mutant mice was reduced to the level found in control mice after ganglionic blockade with chlorisondamine (chlori). Chlorisondamine did not change the glucagonemia of control mice. (C) Plasma glucagon levels measured at the end of 3 hours of hypoglycemic $(\sim 2.5 \mathrm{mM})$ or euglycemic $(\sim 5.5 \mathrm{mM})$ clamps. Hypoglycemia induced an approximately 4-fold increase in glucagon plasma levels in control mice and an approximately 2 -fold increase in mutant mice. (D) Plasma glucagon levels measured 60 minutes after i.p. injections of $\mathrm{NaCl}$ or 2-DG in $\mathrm{C} 57 \mathrm{BI}$ and ripglut1;g/ut2--(B6) mice. 2-DG induced a 5- and 3-fold increase in plasma glucagon in control and mutant mice, respectively. (E) Plasma glucagon levels measured 30 minutes after i.c.v. injection of $\mathrm{NaCl}$ or 2-DG. 2-DG induced a 5-fold increase in plasma glucagon in C57BL/6 mice and an approximately 3-fold increase in mutant mice. Data are indicated as mean $\pm \mathrm{SD} ; n=6-8$ for each data point. (C) Data are indicated as mean \pm SEM of 3 experiments, each performed with 5-6 mice. ${ }^{* *} P<0.01$ and ${ }^{* * *} P<0.005$ for comparison between $\mathrm{NaCl}$ - and 2-DG-injected groups. ${ }^{\#} P<0.05$ and ${ }^{\# \# P}<0.01$ for comparison between $\mathrm{NaCl}$-injected control and ripglut1; glut2-l-(B6) groups (Student's $t$ test).

enzymes, albeit at different levels (42-44). Thus, an alternate explanation for the different hypoglycemia-induced glucagon response observed in the mutant mice with different genetic backgrounds may be related to strain-dependent activation thresholds of the hypoglycemia detection systems.

The presence of different sensors controlling glucagon secretion at different hypoglycemic levels was previously proposed to explain the absence of glucagon secretion at approximately $2.5 \mathrm{mM}$ in ripglut1,glut2-/- mice, which, however, presented a strong response at approximately $1 \mathrm{mM}(29)$. In addition, our data showing that c-FLI-positive cells are increased similarly in the $\mathrm{VMH}$ of control and GLUT2-null mice indicate that this structure is sensitive to a glucoprivic signal independently of the expression of GLUT2. The VMH has been associated with the control of counterregulation; its detection system may therefore be distinct from that of the brainstem. It could be involved in the stimulation of glucagon secretion at deep hypoglycemic levels.

The observation that the same gene mutation can lead to different phenotypes when present in different genetic backgrounds has been reported in many other cases. For instance, transferring the $i r s 1^{+/-} ;$insr $^{+/-}$mutations in 3 different genetic backgrounds (C57BL/6, 129Sv, and DBA) led to insulin resistance but with completely different levels of insulinemia, $\beta$-cell hyperplasia, and diabetes (45). Similarly, the irs $2^{-/-}$mutation in a heterozygous background leads to a strong diabetic phenotype already at 6 weeks after birth (46) whereas, when backcrossed in the C57BL/ 6 background, this mutation leads to a mild glucose intolerance that becomes obvious only after 20 weeks of life (47).
The existence of different classes of cerebral glucose sensors is consistent with the studies of Yang et al. (48), who showed that neuronal glucodetection could be GLUT2 independent. Indeed, in the $\mathrm{VMH}$, only $30 \%$ of glucose-responsive neurons and $22 \%$ of glucose-sensitive neurons express the GLUT2 transporter (49). Furthermore, a number of these glucose-modulated neurons express the sodium-coupled glucose transporter SGLT-1, and pharmacologic inhibition of this transporter by phloridzin suppresses, respectively, electrical activation and inhibition of glucose-responsive and glucose-sensitive neurons (48-50). Also, the majority of glucose-modulated neurons express GLUT3; this transporter may thus be required and sufficient for glucose signaling in these neurons. Unlike neurons, the majority of astrocytes express GLUT1 (51). Because this transporter carries glucose with an affinity around $3 \mathrm{mM}$, it is possible that GLUT1 instead of GLUT2 is involved in cerebral GLUT2-independent glucodetection and control of glucagon secretion in C57BL/6 mice.

Together, our data show that GLUT2 is a critical component of the glucose sensors that regulate fed plasma glucagon levels and that it also participates in sensors controlling hypoglycemia-stimulated glucagon secretion. In these mechanisms, GLUT2 is expressed in glial cells, supporting the hypothesis of a metabolic coupling between astrocytes and neuronal cells in glucodetection. Our data also provide indication that these sensors are part of or activate cells of the NTS and DMNX. Identifying these GLUT2-expressing cells in the brainstem and other CNS sites may lead to a better understanding of the cellular basis for the control of counterregulation in the physiological state and its impairment in diabetes. 


\section{Methods}

Animals. Mice were maintained at $24 \pm 1^{\circ} \mathrm{C}$, with a $12 / 12$ hour light/dark cycle and used when 12 to 14 weeks old. The ripglut1;glut $2^{-1-}$ mice (30) and their ripglut1;glut $2^{+/-}$littermates were maintained in a mixed genetic background. For the indicated experiments, the ripglut1;glut2--- mice were backcrossed into C57BL/6 mice (Janvier) for 7 generations. Animal experimentation was approved by the Office of the Véterinaire Cantonal Vaudois, Lausanne, Switzerland.

Transgenic mice. For expression of GLUT2 in astrocytes, mouse GLUT2 cDNA (52) was first subcloned downstream of the GFAP promoter contained in the pGFGH plasmid (gift of I.L. Campbell, UCSD, La Jolla, California, USA) (53). A full-length mouse GLUT2 cDNA was constructed by ligating a glut2 EcoRI-SalI fragment (nt 1542-1625 cDNA) with the 5 ' end of the mouse GLUT2 cDNA (nt 1-1542). The full-length mouse GLUT2 cDNA was excised by NotI and XhoI digestion, blunted, and subcloned downstream of the GFAP promoter into NotI cut and blunted pGFGH plasmid. The transgene was isolated as a $4.7-\mathrm{kb}$ SfiI-PvuI fragment and injected into ripglut $1^{+-} ;$; $l u t 2^{+/-}$fertilized oocytes obtained by crossing B6D2F1 females with ripglut1 $1^{++}$, glut2 $2^{-/-}$males. The founders (pgfapglut2; ripglut1;glut2+--) were crossed with ripglut1;glut2-/- mice to obtain pgfapglut2; ripglut1;glut2 $2^{-/-}$mice and their control littermates (ripglut1;glut2 $2^{-/}$and ripglut $\left.1 ; g l u t 2^{+-}\right)$. The genetic background of these mice was derived from C57BL/6, Sv129, and B6D2F1 strains.

Transgene detection was by PCR and Southern blot of tail DNA (see Figure 3). For PCR analysis, primers P1 (TTAGTCCAACCCGTTCCT) and G1 (GTGTGGTGGAATTGTCCT) were used to amplify a 420-bp fragment. Through Southern blot analysis, the transgene was detected as a $3.8-\mathrm{kb}$ EcoRI fragment.

For preparation of a transgene for expression of GLUT2 in neurons, a $0.8-\mathrm{kb}$ EcoRI-KpnI restriction fragment derived from $\mathrm{pHD} 2 \mathrm{WOP}$ plasmid and containing the SV-40 polyadenylation signal sequence (SV40-polyA) was blunted and subcloned into the SalI cut and blunted full-length mouse GLUT2 cDNA described above. A 2.6-kb SmaI-KpnI fragment from this plasmid was blunted and subcloned downstream of the synapsin promoter contained in the pBL4.3Syn-CAT plasmid (gift of M.W. Kilimann, University of Bochum, Bochum, Germany) (54), cut with XhoI and SmaI, and blunted. The 7-kb SalI fragment transgene was injected into oocytes as described above. We then generated psynglut2;ripglut1;glut2-/- mice and their control littermates ripglut1;glut2-/and ripglut1;glut2+/.

The transgene was identified by PCR and Southern blot of tail DNA (see Figure 5). For PCR analysis, primers P2 (GAGCCCCTTGCGCTCCGCCC) and G2 (TGGGTACTCTTCACCAAC) generated a 720-bp fragment. Through Southern blot analysis, the transgene was detected as a 3.7-kb HindIII fragment.

Northern and Western blot and immunofluorescence microscopy analysis. Total RNA was extracted (55) and analyzed for GLUT2 mRNA as described (56). GLUT2 Western blot analysis was performed as described (57). Immunofluorescence microscopy detection of GLUT2 and GFAP was performed on frozen sections of $4 \%$ paraformaldehyde, perfusedfixed tissues $(30,57,58)$.

Analytical procedures. Glycemia was measured from tail vein blood using a glucose meter (Roche Diagnostics Corp.). Glucagon levels were quantitated by radioimmunoassay (Linco Research Inc.) in plasma prepared from blood sampled from the retroorbital plexus in the presence of $1 \mu \mathrm{g} / \mathrm{ml}$ aprotinin and $1 \mathrm{mM}$ EDTA.

Intraperitoneal injections. The mice were manipulated daily for 15 days before the experiments. On the test day, their glycemia was measured twice at an interval of 30 minutes. If their glycemia was stable, the mice were injected with a saline solution or 2-DG at $150 \mathrm{mg} / \mathrm{kg}$. Injections were performed between 8:00 and 9:00 am in fed mice. Blood was collected by retroorbital puncture 30 minutes later. When used, chlorisondamine was injected at $5 \mathrm{mg} / \mathrm{kg}$.

Intracerebroventricular injections. For i.c.v. injections, the animals were anesthetized and placed in a stereotactic frame. A stainless steel cannula (30 gauge) was implanted in the right lateral ventricle according to the mouse brain coordinates $(58)(0.5 \mathrm{~mm}$ posterior to the bregma, $1.3 \mathrm{~mm}$ lateral to midline, and $2.1 \mathrm{~mm}$ below the surface of the skull). The animals were then allowed to recover for 2 days. Between 8:00 and 10:00 am on the test day, 2-DG ( $1 \mathrm{mg} /$ mouse) or saline was injected in fed mice. Placement of cannulas was verified by histological analysis of frozen brain sections.

Hyperinsulinemic clamps. The mice were housed with an inverted light cycle with darkness from 8:00 am to 8:00 pm. An indwelling catheter was implanted in mice into the vena cava through the left femoral vein as described (29). The other extremity of this catheter was slid under the back skin, exteriorized, and glued at the back of the neck. The day of the experiment, food was removed at 8:00 am for 6 hours. Human insulin (Actrapid; Novo Nordisk) was infused at the rate of $18 \mathrm{mU} / \mathrm{kg}^{-1} / \mathrm{min}^{-1}$ for 180 minutes, and a $20 \%$ glucose solution was coinfused with insulin to maintain glycemia at 2.5 or $5.5 \mathrm{mM}$.

c-Fos immunohistochemistry. Two hours after the i.p. saline or 2-DG injection, the brains were fixed as described above, and $40 \mathrm{~nm}$ serial frozen sections were prepared. Hypothalamic (from the level of bregma $-0.46 \mathrm{~mm}$ to $-2.30 \mathrm{~mm}$ ) and brainstem (from the level of bregma $-8 \mathrm{~mm}$ to $-6.64 \mathrm{~mm}$ ) sections were preincubated for 30 minutes with BSA $3 \%$ (Sigma-Aldrich), then incubated for 48 hours at $4^{\circ} \mathrm{C}$ with a rabbit polyclonal antiserum to human FOS4-17 (1:10,000, Ab-5; Oncogene Sciences Inc.), diluted in $0.1 \mathrm{M}$ PBS containing $0.3 \%$ Triton X-100 (Sigma-Aldrich) (PBS-TX). The sections were then incubated with goat anti-rabbit immunoglobulinHRP conjugates (1:1.000; DakoCytomation) for 24 hours at room temperature. HRP activity was revealed by 3,3'-diaminobenzidine tetrahydrochloride (DAB; Sigma-Aldrich) reaction. Quantification of c-Fos-positive nuclei was performed bilaterally in $10-15$ sections from each structure and for 6 to 8 mice per condition.

Statistics. Statistical analysis for all experiments was performed using the 2-tailed Student's $t$ test.

\section{Acknowledgments}

We thank Wanda Dolci for performing glucagon radioimmunoassays. This work was supported by grants 3100-065219-01 from the Swiss National Science Foundation and 1-2002-366 from the Juvenile Diabetes Research Foundation International (B. Thorens).

Received for publication July 18, 2005, and accepted in revised form September 27, 2005.

Address correspondence to: Bernard Thorens, Institute of Physiology, Center for Integrative Genomics, University of Lausanne, Génopode Building, CH-1015 Lausanne, Switzerland. Phone: 41-21-69239-81; Fax: 41-21-292-39-85; E-mail: Bernard.Thorens@unil.ch.

Michel Dallaporta's present address is: Laboratoire de physiologie Neuro-Végétative, CASE 351, Université Paul Cézanne, Marseille, France.

Marc Foretz's present address is: Département de Génétique, Développement et Pathologie, Institut Cochin, INSERM, CNRS, Paris, France. 
1. Cryer, P.E., Davis, S.N., and Shamoon, H. 2003. Hypoglycemia in diabetes. Diabetes Care. 26:1902-1912.

2. Cryer, P.E. 2004. Diverse causes of hypoglycemiaassociated autonomic failure in diabetes. N. Engl.J. Med. 350:2272-2279.

3. Weir, G.C., Knowlton, S.D., and Martin, D.B. 1974. Glucagon secretion from the perfused rat pancreas. Studies with glucose and catecholamines. J. Clin. Invest. 54:1403-1412.

4. Pipeleers, D.G., et al. 1985. Interplay of nutrients and hormones in the regulation of insulin release. Endocrinology. 117:824-833.

5. Heimberg, H., et al. 1996. The glucose sensor protein glucokinase is expressed in glucagon-producing $\alpha$ cells. Proc. Natl. Acad. Sci. U. S. A. 93:7036-7041.

6. Suzuki, M., et al. 1999. Immuno-localization of sulphonylurea receptor 1 in rat pancreas. Diabetologia. 42:1204-1211.

7. Heimberg, H., De Vos, A., Pipeleers, D., Thorens, B., and Schuit, F. 1995. Differences in glucose tranporter gene expression between rat pancreatic $\alpha$ - and $\beta$-cells are correlated to differences in glucose transport but not in glucose utilization. J. Biol. Chem. 270:9871-9875.

8. Raju, B., and Cryer, P.E. 2005. Loss of the decrement in intraislet insulin plausibly explains loss of the glucagon response to hypoglycemia in insulin-deficient diabetes: documentation of the intraislet insulin hypothesis in humans. Diabetes. 54:757-764.

9. Gosmanov, N.R., et al. 2005. Role of the decrement in intraislet insulin for the glucagon response to hypoglycemia in humans. Diabetes Care. 28:1124-1131.

10. Ahren, B., Taborsky, G.J., and Porte, D. 1986. Neuropeptidergic versus cholinergic and adrenergic regulation of islet hormone secretion. Diabetologia. 29:827-836

11. Havel, P.J., et al. 1993. Autonomic control of pancreatic polypeptide and glucagon secretion during neuroglucopenia and hypoglycemia in mice. Am. J. Physiol. 265:R246-R254.

12. Thorens, B. 2004. The hepatoportal glucose sensor. Mechanisms of glucose sensing and signal transduction. In Glucokinase and glycemic disease: from basics to novel therapentics. F.M. Matschinski and M.A. Magnuson, editors. Karger. Basel, Switzerland/New York, New York, USA. 327-338.

13. Thorens, B., and Larsen, P.J. 2004. Gut-derived signaling molecules and vagal afferents in the control of glucose and energy homeostasis. Curr. Opin. Clin. Nutr. Metab. Care. 7:471-478.

14. Hamilton-Wessler, M., Bergman, R.N., Halter, J.B., Watanabe, R.M., and Donovan, C.M. 1994. The role of liver glucosensors in the integrated sympathetic response induced by deep hypoglycemia in dogs. Diabetes. 43:1052-1060.

15. Hevener, A.L., Bergman, R.N., and Donovan, C.M. 2000. Portal vein afferents are critical for the sympathoadrenal response to hypoglycemia. Diabetes. 49:8-12.

16. Frizzell, R.T., et al. 1993. Counterregulation during hypoglycemia is directed by widespread brain regions. Diabetes. 42:1253-1261.

17. Borg, M.A., Sherwin, R.S., Borg, W.P., Tamborlane, W.V., and Shulman, G.I. 1997. Local ventromedial hypothalamus glucose perfusion blocks counterregulation during systemic hypoglycemia in awake rats. J. Clin. Invest. 99:361-365.

18. Evans, M.L., et al. 2004. Hypothalamic ATPsensitive $\mathrm{K}+$ channels play a key role in sensing hypoglycemia and triggering counterregulatory epinephrine and glucagon responses. Diabetes. 53:2542-2551.

19. Miki, T., et al. 2001. ATP-sensitive $\mathrm{K}^{+}$channels in the hypothalamus are essential for the maintenance of glucose homeostasis. Nat. Neurosci. 4:507-512.

20. Borg, W.P., Sherwin, R.S., During, M.J., Borg, M.A., and Shulman, G.I. 1995. Local ventromedial hypothalamus glucopenia triggers counterregulatory hormone release. Diabetes. 44:180-184.

21. Ritter, R.C., Slusser, P.G., and Stone, S. 1981. Glucoreceptors controlling feeding and blood glucose: location in the hindbrain. Science. 213:451-453.

22. Ritter, S., Llewellyn-Smith, I., and Dinh, T.T. 1998. Subgroups of hindbrain catecholamine neurons are selectively activated by 2-deoxy-D-glucose induced metabolic challenge. Brain Res. 805:41-54.

23. Ritter, S., Dinh, T.T., and Zhang, Y. 2000. Localization of hindbrain glucoreceptive sites controlling food intake and blood glucose. Brain Res. 856:37-47.

24. Fraley, G.S., and Ritter, S. 2003. Immunolesion of norepinephrine and epinephrine afferents to medial hypothalamus alters basal and 2-deoxyD-glucose-induced neuropeptide $\mathrm{Y}$ and agoutigene-related protein messenger ribonucleic acid expression in the arcuate nucleus. Endocrinology. 411:75-83.

25. Ritter, S., Bugarith, K., and Dinh, T.T. 2001. Immunotoxic destruction of distinct catecholamine subgroups produces selective impairment of glucoregulatory responses and neuronal activation. J. Comp. Neurol. 43:197-216.

26. Dallaporta, M., Himmi, T., Perrin, J., and Orsini, J.-C. 1999. A solitary tract nucleus sensitivity to moderate changes in glucose level. Neuroreport. 10:1-4.

27. Dallaporta, M., Perrin, J., and Orsini, J.-C. 2001. Involvement of adenosine triphosphate-sensitive $\mathrm{K}+$ channels in glucose-sensing in the rat solitary tract nucleus. Neurosci. Lett. 278:77-80.

28. Thorens, B. 2003. A gene knockout approach in mice to identify glucose sensors controlling glucose homeostasis. Pflügers Arch. 445:482-490.

29. Burcelin, R., and Thorens, B. 2001. Evidence that extrapancreatic GLUT2-dependent glucose sensors control glucagon secretion. Diabetes. 50:1282-1289.

30. Thorens, B., Guillam, M.-T., Beermann, F., Burcelin, R., and Jaquet, M. 2000. Transgenic reexpression of Glut 1 or Glut 2 in pancreatic $\beta$ cells rescues Glut2-null mice from early death and restores normal glucose-stimulated insulin secretion. J. Biol. Chem. 275:23751-23758.

31. Silver, I.A., and Erecinska, M. 1998. Glucoseinduced intracellular ion changes in sugar-sensitive hypothalamic neurons. J. Neurophysiol. 79:1733-1745.

32. Routh, V.H. 2002. Glucose-sensing neurons: are they physiologically relevant? Physiol. Behav. 76:403-413.

33. Magistretti, P.J., Pellerin, L., Rothman, D.L., and Shulman, R.G. 1999. Energy on demand. Science. 283:496-497.

34. Arluison, M., et al. 2004. Distribution and anatomical localization of the glucose transporter 2 (GLUT2) in the adult rat brain-an immunohistochemical study. J. Chem. Neuroanat. 28:117-136.

35. Leloup, C., et al. 1994. Glucose transporter 2 (GLUT2): expression in specific brain nuclei. Brain Res. 638:221-226.

36. Jansen, A.S.P., Hoffman, J.L., and Loewy, A.D. 1997. CNS sites involved in sympathetic and parasympathetic control of the pancreas: a viral tracing study. Brain Res. 766:29-38.

37. Arluison, M., Quignon, M., Thorens, B., Leloup, C., and Penicaud, L. 2004. Immunocytochemical localization of the glucose transporter 2 (GLUT2) in the adult rat brain. II. Electron microscopic study. J. Chem. Neuroanat. 28:137-146.

38. Jetton, T.L., et al. 1994. Analysis of upstream glucokinase promoter activity in transgenic mice and identification of glucokinase in rare neuroendocrine cells in the brain and gut. J. Biol. Chem. 269:3641-3654.

39. Maekawa, F., et al. 2000. Localization of glucokinase-like immunoreactivity in the rat lower brain stem: for possible location of brain glucose-sensing mechanisms. Endocrinology. 141:375-384

40. Young, J.K., Baker, J.H., and Montes, M.I. 2000. The brain response to 2-deoxy glucose is blocked by a glial drug. Pharmacol. Biochem. Behav. 67:233-239.

41. Guillod-Maximin, E., Lorsignol, A., Alquier, T., and Penicaud, L. 2004. Acute intracarotid glucose injection towards the brain induces specific c-fos activation in hypothalamic nuclei: involvement of astrocytes in cerebral glucose-sensing in rats. J. Neuroendocrinol. 16:464-471.

42. Wencel, H.E., et al. 1995. Impaired second phase insulin response of diabetes-prone C57BL/6J mouse islets. Physiol. Behav. 57:1215-1220.

43. Kooptiwut, S., et al. 2002. Comparison of insulin secretory function in two mouse models with different susceptibility to beta-cell failure. Endocrinology. 143:2085-2092.

44. Goren, H.J., Kulkarni, R.N., and Kahn, C.R. 2004. Glucose homeostasis and tissue transcript content of insulin signaling intermediates in four inbred strains of mice: C57BL/6, C57BLKS/6, DBA/2, and 129X1. Endocrinology. 145:3307-3323.

45. Kulkarni, R.N., et al. 2003. Impact of genetic background on development of hyperinsulinemia and diabetes in insulin receptor/insulin receptor substrate-1 double heterozygous mice. Diabetes. 52:1528-1534.

46. Withers, D.J., et al. 1998. Disruption of IRS-2 causes type 2 diabetes in mice. Nature. 391:900-904.

47. Suzuki, R., et al. 2003. Pdx1 expression in Irs2 deficient mouse beta-cells is regulated in a strain-dependent manner. J. Biol. Chem. 278:43691-43698.

48. Yang, X.-J., Kow, L.-M., Funabashi, T., and Mobbs, C.V. 1999. Hypothalamic glucose sensor. Similarities to and differences from pancreatic $\beta$ cell mechanisms. Diabetes. 48:1763-1772.

49. Kang, L., Routh, V.H., Kuzhikandathil, E.V., Gaspers, L.D., and Levin, B.E. 2004. Physiological and molecular characteristics of rat hypothalamic ventromedial nucleus glucosensing neurons. Diabetes. 53:549-559.

50. Yang, X.J., Low, L.M., Pfaff, D.W., and Mobbs, C.V. 2004. Metabolic pathways that mediate inhibition of hypothalamic neurons by glucose. Diabetes. 53:67-73.

51. Yu, S., and Ding, W.G. 1998. The $45 \mathrm{kDa}$ form of glucose transporter 1 (GLUT1) is localized in oligodendrocyte and astrocyte but not in microglia in the rat brain. Brain Res. 797:65-72.

52. Suzue, K., Lodish, H.F., and Thorens, B. 1989. Sequence of the mouse liver glucose transporter. Nucleic Acid Res. 17:10099.

53. Stalder, A.K., et al. 1998. Late-onset chronic inflammatory encephalopathy in immune-competent and severe combined immune-deficient (SCID) mice with astrocyte-targeted expression of tumor necrosis factor. Am. J. Pathol. 153:767-783.

54. Hoesche, C., Sauerwald, A., Veh, R.W., Krippl, B., and Kilimann, M.W. 1993. The 5 '-flanking region of the rat synapsin I gene directs neuronspecific and developmentally regulated reporter gene expression in transgenic mice. J. Biol. Chem. 268:26494-26502.

55. Chomczynski, P., and Sacchi, N. 1987. Single-step method of RNA isolation by acid guanidinium thiocyanate-phenol-chloroform extraction. Anal. Biochem. 162:156-159.

56. Gremlich, S., Bonny, C., Waeber, G., and Thorens, B. 1997. Fatty acids decrease IDX-1 expression in rat pancreatic islets and reduce GLUT2, glucokinase, insulin, and somatostatin levels. J. Biol. Chem. 272:30261-30269.

57. Uldry, M., et al. 2001. Identification of a mammalian $\mathrm{H}+$-myo-inositol symporter expressed predominantly in the brain. EMBO J. 20:4467-4477.

58. Paxinos, G., and Watson, C. 1982 . The rat brain in stereotaxic coordinates. Academic Press. New York, New York, USA. 153 pp. 